Article

\title{
Urolithin Metabotypes Can Determine the Modulation of Gut Microbiota in Healthy Individuals by Tracking Walnuts Consumption over Three Days
}

\author{
Izaskun García-Mantrana ${ }^{1, *(1)}$, Marta Calatayud ${ }^{2}\left(\mathbb{D}\right.$, María Romo-Vaquero ${ }^{3}{ }^{(D)}$, \\ Juan Carlos Espín ${ }^{3}\left(\mathbb{D}\right.$, María V. Selma ${ }^{3}$ and María Carmen Collado ${ }^{1, *(\mathbb{D}}$ \\ 1 Department of Biotechnology, Institute of Agrochemistry and Food Technology, IATA-CSIC, \\ 46980 Valencia, Spain \\ 2 Department of Biotechnology, Center for Microbial Ecology and Technology (CMET), Ghent University, \\ 9000 Gent, Belgium; marta.calatayudarroyo@ugent.be \\ 3 Laboratory of Food \& Health, Research Group on Quality, Safety and Bioactivity of Plant Foods, \\ CEBAS-CSIC, 30100 Murcia, Spain; mrvaquero@cebas.csic.es (M.R.-V.); jcespin@cebas.csic.es (J.C.E.); \\ mvselma@cebas.csic.es (M.V.S.) \\ * Correspondence: igama@iata.csic.es (I.G.-M.); mcolam@iata.csic.es (M.C.C.)
}

Received: 12 September 2019; Accepted: 10 October 2019; Published: 16 October 2019

\begin{abstract}
Walnuts are rich in polyphenols ellagitannins, modulate gut microbiota (GM), and exert health benefits after long-term consumption. The metabolism of ellagitannins to urolithins via GM depends on urolithin metabotypes (UM-A, -B, or -0), which have been reported to predict host responsiveness to a polyphenol-rich intervention. This study aims to assess whether UMs were associated with differential GM modulation after short-term walnut consumption. In this study, 27 healthy individuals consumed $33 \mathrm{~g}$ of peeled raw walnuts over three days. GM profiling was determined using $16 \mathrm{~S}$ rRNA illumina sequencing and specific real-time quantitative polymerase chain reactions (qPCRs), as well as microbial activity using short-chain fatty acids analysis in stool samples. UMs stratification of volunteers was assessed using ultra performance liquid chromatography-electro spray ionization-quadrupole time of flight-mass spectrometry (UPLC-ESI-QTOF-MS) analysis of urolithins in urine samples. The gut microbiota associated with UM-B was more sensitive to the walnut intervention. Blautia, Bifidobacterium, and members of the Coriobacteriaceae family, including Gordonibacter, increased exclusively in UM-B subjects, while some members of the Lachnospiraceae family decreased in UM-A individuals. Coprococcus and Collinsella increased in both UMs and higher acetate and propionate production resulted after walnuts intake. Our results show that walnuts consumption after only three days modulates GM in a urolithin metabotype-depending manner and increases the production of short-chain fatty acids (SCFA).
\end{abstract}

Keywords: walnuts; polyphenol; urolithins; metabotypes; gut microbiota; Gordonibacter; personalised nutrition

\section{Introduction}

Nuts are a significant source of macronutrients (e.g., proteins, unsaturated fats, polysaccharides, fiber), micronutrients (e.g., vitamins and minerals), and bioactive compounds (e.g., tocopherols, phytosterols, phenolic compounds [1]). Ellagitannins (ETs) and ellagic acid (EA) are polyphenols present in nuts, pomegranates, and berries [2]. The human gut microbiota can metabolize ETs and EA into the bioactive metabolites urolithins by lactone-ring cleavage, decarboxylation, and dehydroxylation reactions [3]. Epidemiologic studies have associated long-term nut consumption with a reduced incidence of coronary heart disease, hypertension, and cholesterol-lowering effect [4]. The health-related 
effects of nut consumption may be linked to gut microbiota, which can release bioactive compounds with higher bioavailability and functionality than parental compounds [5]. Several studies have demonstrated changes in gut microbial communities after the intake of ETs-containing foods on week timescale [2,6-10]. Besides, some of these studies revealed a personalized effect of foods containing ETs [6], which supports the concept of personalized nutrition. According to how ETs metabolize via gut microbiota, individuals can be stratified into three urolithin metabotypes (UMs) [11,12]. Metabotype A (UM-A) is characterized by the production of urolithin-A (Uro-A). Metabotype B (UM-B) is characterized by the production of urolithin-B (Uro-B), isourolithin-A (IsoUro-A), and Uro-A. Metabotype 0 (UM-0) does not produce these final urolithins [11]. Recently, three urolithin-producing bacteria belonging to the genus Gordonibacter (G. pamelaeae and G. urolithinfaciens) and Ellagibacter isourolithinifaciens were isolated from human feces [13-16]. UMs have been proposed as potential cardiovascular disease (CVD) risk biomarkers [17]. Furthermore, UMs were associated with the host blood lipid profile and with the interindividual variability in the improvement of cardiovascular risk biomarkers after the consumption of pomegranate ellagitannins for six weeks $[6,17]$. In this scenario, this study aims to evaluate the impact of a short-term (three days) nutritional intervention with walnuts on the gut microbiota composition and function in healthy individuals according to their UMs.

\section{Materials and Methods}

\subsection{Study Design and Participants}

A total of 27 healthy individuals (Valencia, Spain) were enrolled in the study. The inclusion criteria were for participants to be older than 18 years old, have a non-declared pathology, and not have taken antibiotics, medication, or pre/probiotics in the two months before the study. The intervention consisted of an intake of $33 \mathrm{~g}$ of walnuts per day for three days. A total of $100 \mathrm{~g}$ of peeled walnuts provided with $65 \mathrm{~g}$ of fats (SFA: $6.4 \mathrm{~g}$; MUFA: $10 \mathrm{~g}$; PUFA: $48 \mathrm{~g}$ ), $4 \mathrm{~g}$ of carbohydrates, $15 \mathrm{~g}$ of protein, $12 \mathrm{~g}$ of dietary fiber, and $0.02 \mathrm{~g}$ of salt. Proanthocyanidins were quantified after acid-catalysis in the presence of phloroglucinol, as previously reported [18]. The analysis of hydrolysable tannins was performed after an acid hydrolysis of nut samples using a method previously reported [19].

Walnut samples contained ( $\mathrm{mg} / \mathrm{g}$ fresh weight; mean $\pm \mathrm{SD}$ ): ellagic acid $4.15 \pm 0.60$; gallic acid $0.48 \pm 0.07$; and catechin $0.44 \pm 0.04$. At the beginning (T0) and the end (T3) of these time-points, participants collected fecal samples for gut microbiota analysis and short fatty acids (SCFA) determination and urine for urolithin determination and subsequent UMs stratification. Moreover, each participant filled out a questionnaire about their clinical, anthropometric, and nutritional records at the end of the interventional study. Additionally, the intake of ellagitannins-containing food, as pomegranate and berries were restricted throughout the intervention study. BMI was calculated and stratified as following: lean-normal weight $\left(\leq 25.0 \mathrm{~kg} / \mathrm{m}^{2}\right)$, overweight $\left(25.0-30.0 \mathrm{~kg} / \mathrm{m}^{2}\right)$, and obese $\left(\geq 30.0 \mathrm{~kg} / \mathrm{m}^{2}\right)$ [20]. Written informed consent was obtained from all the volunteers. The local ethics committee of Atención Primaria-Generalitat Valenciana (CEIC-APCV) approved the study protocol. All experiments were carried out following approved guidelines and regulations.

\subsection{Dietary Estimation}

The dietary intake of the participants was determined through a comprehensive 140-item validated food frequency questionnaire (FFQ) [21]. In all cases, we validated the FFQ information registered by participants with a three-day food record questionnaire for the intake of dietary nutrients. FFQ records were transformed to energy, macro, and micronutrients daily intake using the nutrient food composition tables developed by the Centro de Enseñanza Superior de Nutrición Humana y Dietética (CESNID) and analyzed by EASY DIET software. Moreover, a 14-item, PREDIMED (PREvención con DIeta MEDiterránea) validated test was used to appraise adherence of participants to the Mediterranean $\operatorname{diet}[22]$. 


\subsection{Biological Samples}

Each volunteer collected urine and fecal samples at home using plastic containers following standardized protocol given by the team. Then, those were placed in the freezer at $-20^{\circ} \mathrm{C}$ overnight, before sending to the laboratory where the samples were stored at $-80^{\circ} \mathrm{C}$ until analysis.

\subsection{Urolithin Quantification in Urine}

Urolithins were identified in urine samples after the three-day nut intervention using ultra performance liquid chromatography-electro spray ionization-quadrupole time of flight-mass spectrometry (UPLC-ESI-QTOF-MS), as previously described [23]. The individuals' stratification, according to their UMs (UM-A, UM-B, and UM-0), was carried out as previously described [24].

\subsection{Gut Microbiota Composition Analysis}

DNA was extracted from fecal samples using a commercial kit, the Master-Pure DNA Extraction Kit (Epicentre, Madison, WI, USA), following the manufacturer's instructions with modifications described in García-Mantrana [25]. Purification of the DNA was performed using the DNA Purification Kit (Macherey-Nagel, Duren, Germany) according to the manufacturer's instructions.

Specific primers (F_Gordon and R_Gordon) and a TaqMan probe (P_Gordon) targeting the $16 S$ rRNA gene of Gordonibacter genus were used for quantification via the real-time quantitative polymerase chain reactions (qPCR) [26], because Gordonibacter genus appears in a very low relative abundance, and is not possible to detect by next-generation sequencing (NGS) methods. A touch-down qPCR protocol was applied for DNA amplification and the ramping profile was: 1 cycle at $95^{\circ} \mathrm{C}$ for $5 \mathrm{~min}$, followed by the first 20 cycles of $94^{\circ} \mathrm{C}$ for $30 \mathrm{~s}$, an auto-increment of $0.5^{\circ} \mathrm{C}$ from 55 to $65^{\circ} \mathrm{C}$ for $45 \mathrm{~s}, 72{ }^{\circ} \mathrm{C}$ for $2 \mathrm{~min}$. Then, the second 20 cycles of $94{ }^{\circ} \mathrm{C}$ for $30 \mathrm{~s}, 55^{\circ} \mathrm{C}$ for $45 \mathrm{~s}$, and $72{ }^{\circ} \mathrm{C}$ for $2 \mathrm{~min}$ were applied. Finally, it was added 1 cycle at $72{ }^{\circ} \mathrm{C}$ for $5 \mathrm{~min}$.

Gut microbiota composition and diversity were determined by V3-V4 variable region of the $16 \mathrm{~S}$ rRNA gene sequencing, following Illumina protocols. After $16 \mathrm{~S}$ rDNA gene amplification, Nextera XT Index Kit (Illumina, San Diego, CA, USA) was used for the multiplexing step and a Bioanalyzer DNA 1000 chip (Agilent Technologies, Palo Alto, CA, USA) for checking the PCR product quality. Libraries were sequenced using a $2 \times 300 \mathrm{pb}$ paired-end run (MiSeq Reagent kit v3) on a MiSeq-Illumina platform (FISABIO sequencing service, Valencia, Spain), according to the manufacturer's instructions (Illumina). Statistical analyses of the 16S rRNA gene sequence data were performed with QIIME statistical tools [27]. Chimeric sequences and sequences that could not be aligned were removed from the data set. Sequences that were classified as Cyanobacteria and Chloroplasts were also removed.

\subsection{Statistical Analysis}

Calypso software version 8.84 was used with total sum normalization (TSS) for data mining, multivariate testing, including a redundancy analysis (RDA) and parametric tests [28]. Subsequently, beta diversity based on the Bray Curtis distance (non-phylogenetic) was carried out. To test significance between groups, PERMANOVA test was used. Alpha diversity indexes (Chao1, Simpson, and Shannon) were also determined. Linear discriminant analysis effect size (LEfSe) was used to detect bacterial features between metabotypes and conditions. Data were classified by metadata factors. Graphpad Prism v. 5.04 was used for $t$-test analysis according to data normality assessed by Kolmogorov-Smirnov and Shapiro-Wilk test. Pearson correlations between relative abundances of bacterial groups and SCFA levels were done using Calypso software. Data were considered statistically significant at $p<0.05$.

\subsection{Gut Microbiota Activity Analysis}

Fecal supernatants were obtained from $100 \mathrm{mg}$ of fecal sample in $1 \mathrm{~mL}$ of buffered saline solution. Short Chain Fatty Acids (SCFA) analysis was carried out in the fecal supernatants that were filtered 
through $0.45 \mu \mathrm{m}$-pore-size nitrocellulose filters (Millipore, Burlington, MA, USA). C2-C8 fatty acids analysis was performed as described [29].

\section{Results}

\subsection{Characteristics of Subjects}

The analysis of urolithins in urine after three days of walnut consumption was used for clustering the individuals according to their UMs: UM-A $(n=14), \mathrm{UM}-\mathrm{B}(n=13)$, and UM-0 $(n=0)$. No significant differences were found in the demographic, clinical, and nutritional characteristics, as well as the Mediterranean diet score according to UMs (Table 1).

Table 1. Clinical and nutritional characteristics of the participants.

\begin{tabular}{lcccc}
\hline & \multicolumn{4}{c}{ Urolithin Metabotypes } \\
\hline & Total $(n=\mathbf{2 7})$ & UM-A $(\boldsymbol{n}=\mathbf{1 4})$ & UM-B $(\boldsymbol{n}=\mathbf{1 3})$ & $\boldsymbol{p}$ Value \\
\hline Age (years) & $39.5 \pm 7.3$ & $36.1 \pm 7.2$ & $43.1 \pm 5.6$ & 0.521 \\
BMI (kg/cm ${ }^{2}$ ) & $23.3 \pm 3.2$ & $23.9 \pm 3.5$ & $22.7 \pm 2.8$ & 0.625 \\
$\quad$ Normal Weight (\%) & 70.4 & 64.3 & 76.9 & 0.961 \\
Overweight (\%) & 29.6 & 35.7 & 23.1 & 0.393 \\
Sex & & & & \\
$\quad$ Female (\%) & 55.5 & 57.1 & 61.5 & 0.471 \\
Male (\%) & 40.7 & 42.8 & 38.5 & 0.565 \\
MD (\%) & 55.5 & 57.1 & 53.8 & 0.669 \\
Total Protein (g/day) & $93.9 \pm 15.5$ & $95.5 \pm 13.5$ & $92.2 \pm 17.9$ & 0.597 \\
$\quad$ Animal-Derived Protein (g/day) & $47.7 \pm 13.9$ & $50.8 \pm 12.4$ & $44.4 \pm 15.1$ & 0.237 \\
Plant-Derived Protein (g/day) & $44.1 \pm 7.7$ & $42.4 \pm 7.4$ & $45.9 \pm 7.9$ & 0.232 \\
Lipids (g/day) & $78.8 \pm 9.5$ & $80.9 \pm 8.6$ & $76.5 \pm 10.3$ & 0.246 \\
$\quad$ SFA & $16.5 \pm 3.2$ & $16.1 \pm 3.5$ & $16.9 \pm 3.0$ & 0.527 \\
MUFA & $33.5 \pm 4.9$ & $33.7 \pm 5.4$ & $33.3 \pm 4.5$ & 0.844 \\
PUFA & $12.8 \pm 2.5$ & $12.6 \pm 2.1$ & $13.0 \pm 2.9$ & 0.684 \\
Total Carbohydrates (g/day) & $215.4 \pm 29.7$ & $207.8 \pm 24.3$ & $223.6 \pm 33.6$ & 0.171 \\
Dietary Fiber (g/day) & $26.1 \pm 5.9$ & $26.7 \pm 6.4$ & $25.4 \pm 5.7$ & 0.593 \\
$\quad$ Insoluble Dietary Fiber (g/day) & $16.8 \pm 4.8$ & $17.2 \pm 4.9$ & $16.3 \pm 4.8$ & 0.636 \\
$\quad$ Soluble Dietary Fiber (g/day) & $3.1 \pm 0.8$ & $3.0 \pm 0.9$ & $3.2 \pm 0.8$ & 0.553 \\
\hline
\end{tabular}

Results are presented as mean \pm SD and percentage (\%). SFA: saturated fatty acids; MUFA: monounsaturated fatty acids; PUFA: polyunsaturated fatty acids. $p<0.05$ for comparison the clinical and nutritional characteristics between UMs. MD: Mediterranean diet adherence.

\subsection{Baseline Microbiota Composition is Urolithin-Metabotype Dependent}

Baseline microbiota (T0, $n=27)$ was characterized by the presence of Firmicutes with a mean relative abundance and SD of $77.31 \% \pm 2.88$, followed by Bacteroidetes $(15.86 \% \pm 0.28)$, Actinobacteria $(3.13 \% \pm 0.65)$, Verrucomicrobia $(1.78 \% \pm 1.22)$, and Proteobacteria $(1.31 \% \pm 1.50)$ at the phylum level. The relative abundance of phylum Synergistetes was significantly enriched in UM-B $(p<0.05)$ compared to UM-A. At the family level, the family Lachnospiraceae was associated with UM-A, whereas the abundance of the families Cerasicoccaceae, Peptostreptococcaceae, Synergistaceae, and Paraprevottelaceae were related to UM-B. A redundancy analysis (RDA) on genus level showed significant differences in the microbial population, according to UMs $(p=0.025)$ (Figure 1A). Volunteers belonging to UM-B had a higher richness ( $p=0.003$, Chao 1 index) than UM-A (Figure 1B).

Lefse analysis showed that Dorea, Holdemania genus, and members of Lachnospiraceae family were enriched in UM-A, while genus cc_115, Oxalobacter, members of Synergistaceae, Cerasicoccaceae, Coriobacteriacea, Peptostreptococcaceae family, and Paraprevotella were associated with UM-B individuals (Figure 1C). 

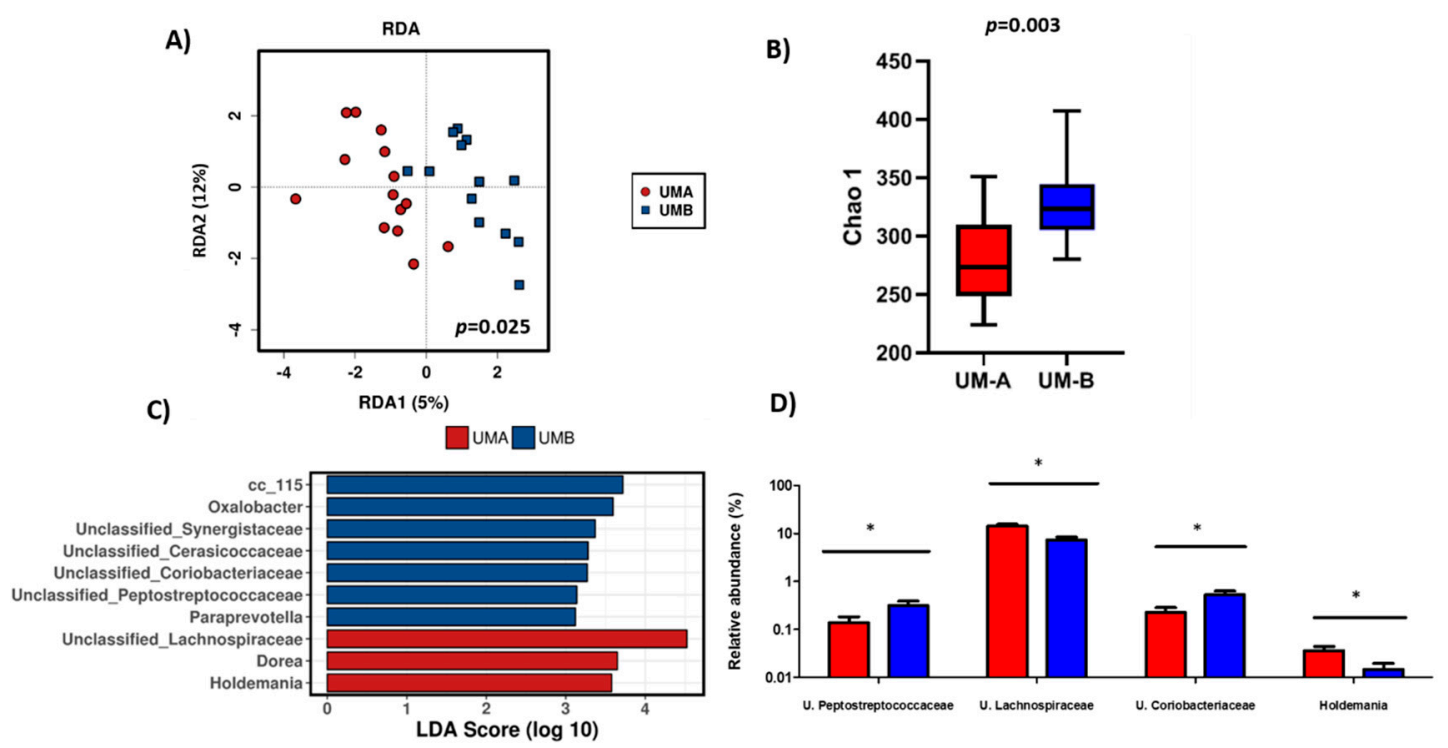

Figure 1. Gut microbiota composition and diversity according to metabotypes (UM-A and UM-B) before the intervention (T0). (A) Redundancy analysis (RDA) on the genus level. (B) Gut microbial richness was measured by Chao index. (C) Linear discriminant analysis (LDA) effect size (LEfSe) plot of taxonomic biomarkers identified in the gut microbiome of volunteers. (D) Relative abundances on genus level in UM-A and UM-B. ANOVA test was used to test significance differences in the relative abundances according to metabotype. Results are presented as mean \pm SD. Significant differences $(p<0.05)$ are marked with an asterisk $\left.{ }^{*}\right)$.

\subsection{Microbiota Composition of UM-B is Sensitive to Walnuts Consumption}

The redundancy analysis (RDA) of all individuals $(n=27)$ showed that bacterial communities were significantly affected by the three day walnut intervention $(p=0.001)$. However, when the volunteers were stratified according to their UMs, these shifts were only significant for UM-B ( $p=0.017)$ (Figure 2D). At the phylum level, walnut intake decreased the relative abundance of Bacteroidetes and increased Actinobacteria when all individuals were considered $(p<0.05)$. These differences were consistent for UM-B after clustering. At the family level, walnut consumption increased the Coriobacteriaceae family in both UMs, while the Bifidobacteriaceae family increased after three days only for UM-B volunteers. Among bacterial genera, members of the Lachnospiraceae family decreased after the walnut intervention in UM-A individuals, Coprococcus and Collinsella increased in both UMs, whereas Blautia, Bifidobacterium, and unclassified Coriobacteriaceae increased only in participants belonging to UM-B $(p<0.05)$ (Figure 2B,E). According to the Chao1 index, gut microbial richness significantly decreased for UM-A $(p=0.024)$ and UM-B $(p<0.001)$ after walnut consumption (Figure $2 \mathrm{C}, \mathrm{F})$. 
A)

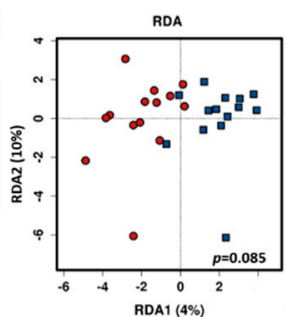

B)

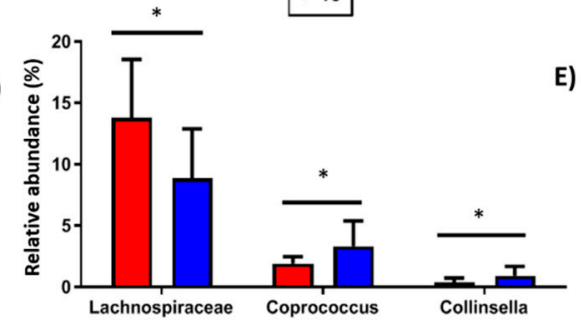

C)

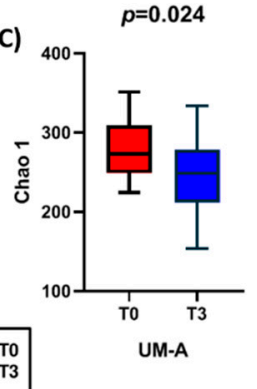

D)

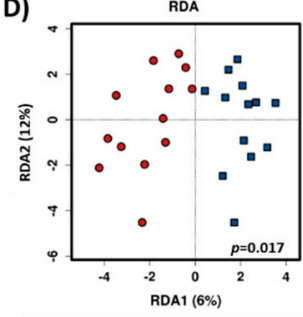

F)

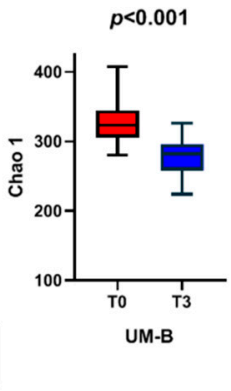

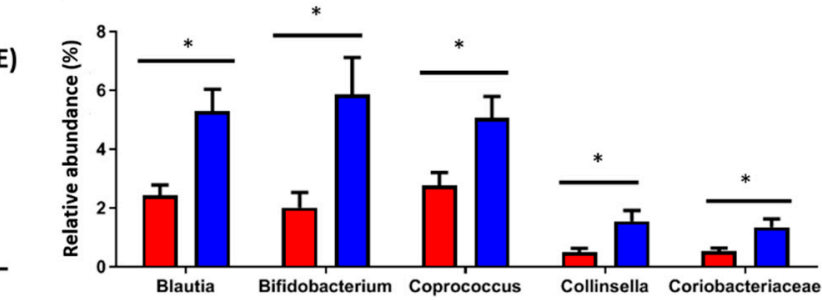

Figure 2. Effect of the intervention with walnuts in gut microbiota composition $(\mathbf{A}, \mathbf{B})$ and diversity $(\mathbf{C})$ in UM-A and in gut microbiota composition $(\mathbf{D}, \mathbf{E})$ and diversity $(\mathbf{F})$ in UM-B. ANOVA test was used to test significance differences in the relative abundances before and after the intervention. Alpha diversity measured by Chao1 index. Results are presented as mean \pm SD. Significant differences $(p<0.05)$ are marked with an asterisk $\left({ }^{*}\right)$.

\subsection{Walnut Consumption Increased Faecal Gordonibacter Levels in UM-B}

Before the intervention, UM-A tended to present higher counts of Gordonibacter. However, it was not significant compared to UM-B $(p=0.601)$ (Figure 3A). After intervening and analyzing the individuals as a single group, a significant increase of fecal Gordonibacter levels was observed $(p=0.024)$ (Figure 3B). After clustering, according to UMs, walnut consumption only increased Gordonibacter levels in UM-B individuals $(p=0.034$ ) (Figure 3C,D). No significant associations with BMI, MD stratification, nutrient intake, or sex with Gordonibacter levels were found.

A)

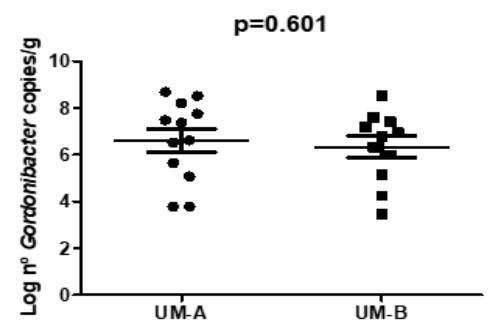

C)

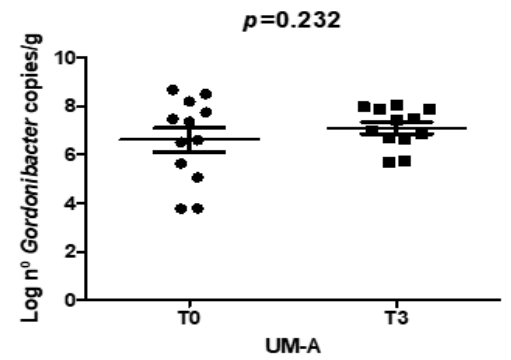

B)

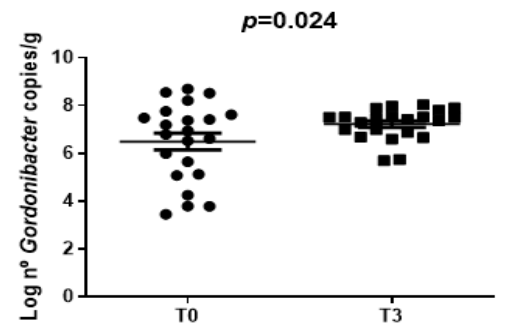

D)

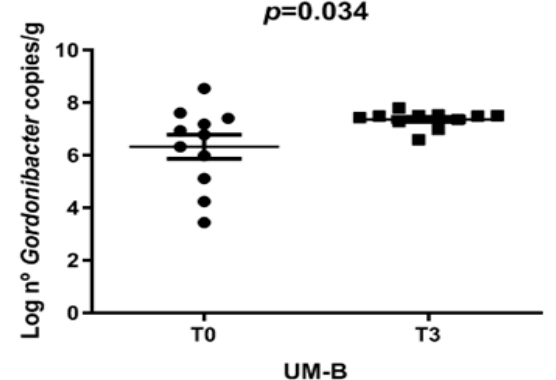

Figure 3. Microbiota composition before (T0) and after the intervention (T3). Gordonibacter levels measured using qPCR according to UMs before the intervention (A). Gordonibacter levels before and after the intervention for all the participants (B). Gordonibacter levels before and after the intervention for UM-A (C) and UM-B participants (D). Paired t-test was used to test significance differences in Gordonibacter levels before and after the intervention. Results are presented as mean \pm SEM. 


\subsection{Effect of Walnut Consumption on Gut Microbiota Activity}

Before the intervention, volunteers belonging to UM-A tended to present higher levels of butyrate $(p=0.070)$. After the intervention, we did not find significant differences in SCFA production after walnut consumption according to UMs (data not shown), but analyzing all participants as a single group, the walnut intake increased the total SCFA $(p=0.012)$ levels, specifically acetate $(p=0.021)$ and propionate $(p=0.040)$ (Figure 4$)$.

A)

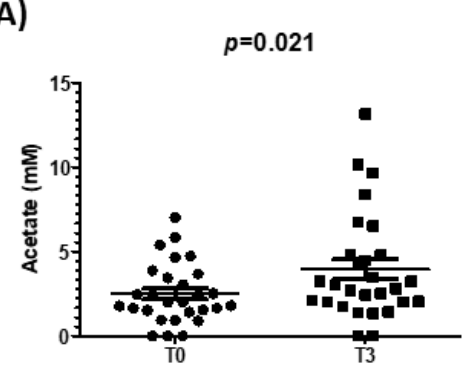

C)

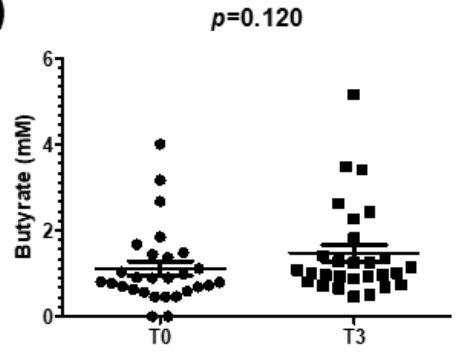

B)

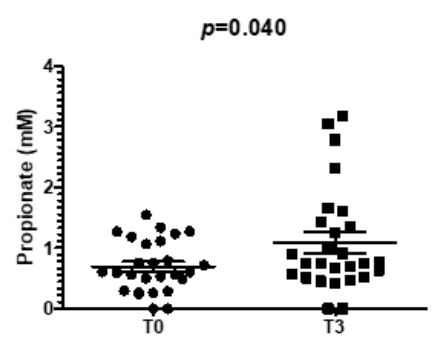

D)

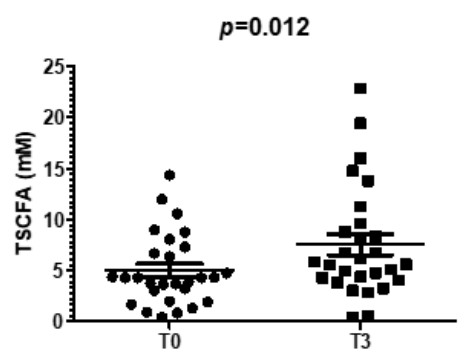

Figure 4. SCFA profile before and after the intervention for all the participants. Acetate (A), propionate (B), butyrate (C) and total SCFA (D) levels measured by GC for all the participants. A paired t-test was used to test significant differences in SCFA levels before and after the intervention. Results are presented as mean \pm SEM.

\subsection{Associations between Gut Microbiota Composition/Activity and Walnut Intervention}

Before the walnut intervention, at the genus level a positive correlation was found between the increase in acetate and Phascolarctobacterium $(p=0.006)$ and Anaerostipes $(p=0.011)$, propionate and Phascolarctobacterium $(p=0.040)$, Coprococcus $(p=0.031)$ and Anaerostipes $(p=0.011)$, and butyrate and Coprococus ( $p=0.030$ ) and Anaerostipes $(p=0.020)$, respectively. At the species level, we found that this positive correlation between propionate and butyrate with Coprococus was due to Coprococcus catus. After the walnut intervention, positive correlations were observed between acetate levels and Phascolarctobacterium ( $p=0.018$ ) and Dehalobacterium $(p=0.014)$, propionate levels and Eubacterium $(p=0.024)$, Phascolarctobacterium $(p=0.040)$, Slackia $(p=0.024)$, and butyrate levels with Dehalobacterium $(p=0.016)$, and Eubacterium $(p=0.006)$.

\section{Discussion and Conclusions}

Understanding individuals' response to dietary bioactive compounds such as polyphenols is essential in the context of personalized nutrition [30]. The occurrence of specific gut microbiota metabotypes can affect the metabolism and bioactivity of polyphenols and the response of individuals upon polyphenol consumption can vary [6]. Our present study shows that the impact exerted by the three day walnut intervention on the gut microbiota depends on the urolithin metabotypes (UMs) of healthy individuals. Therefore, our results add significance to previous studies that aimed to explain the controversial benefits of bioactive compounds present in foods due to the inherent high inter-individual variability [6]. Notably, only UM-B participants responded significantly to the intervention with walnuts towards a change in the gut microbiota composition. 
Parameters such as baseline gut microbiota composition and habitual daily intakes may influence a differential host response [31]. Furthermore, gut microbiota composition would be considered as a predictor for the inter-individual variability in response to dietary interventions [31]. Although UM groups did not present differences in their dietary intakes, significant differences in the gut microbial composition, according to UMs clustering, were observed. In agreement with another study [32], higher relative abundances of Synergistetes phylum and members of Coriobacteriaceae family were observed in UM-B individuals, whereas members of the Lachnospiraceae family predominated in UM-A. We also found a higher gut microbiota richness in UM-B individuals in agreement with Romo-Vaquero and colleagues [32]. Differences of basal microbiota in UMs may be linked to the individual response to ETs intake. It has been reported that intervention with an ellagitannin-rich pomegranate extract for three weeks exerted a differential impact on cardiovascular risk markers of individuals depending on their UM metabotype [6]. The cardiovascular benefits were mainly observed in UM-B individuals, who initially presented a higher cardiovascular risk [6]. These results are in agreement with our present study, where only UM-B subjects responded significantly to the walnut intervention. Moreover, a previous study that evaluated the capacity to produce nut phenolic metabolites in subjects with metabolic syndrome showed that several urolithins significantly increased after a nut-enriched-diet. However, this pathology is associated with an altered gut bacterial diversity [33].

Overall, the evidence on the impact of walnuts on the gut microbiota is scarce and mainly provided by long-term interventions where the significance of the gut microbiota metabotypes, linked to the inter-individual variability, was not explored $[8,9,34]$. In agreement with González-Sarrías et al., our results supported the personalized effect associated with ellagitannin-containing foods consumption [6]. In the present study, a three day walnut intervention modulated some microbial groups, including the increase of Blautia, Bifidobacterium and bacterial members of Coriobacteriaceae, such as Gordonibacter, but only in UM-B individuals. Previous research has shown that walnut consumption increased the relative abundance of butyrate-producing species, although the authors observed opposite results on some bacterial groups such as Ruminococcus and Bifidobacteria [8,35]. Holscher et al. assessed the impact of consuming $42 \mathrm{~g}$ walnuts per day for two three week periods on the human gut microbiota composition and observed a decrease in the relative abundance of Ruminococcus and Bifidobacteria [8]. However, another recent study showed that the same amount of walnuts per day enhanced the abundance of Ruminococcaceae and Bifidobacteria [35]. The effect of walnut consumption on the gut microbial composition may be linked to ellagic acid and ellagitannins, but other polyphenols and other compounds such as dietary fiber and unsaturated fats [4] would also be influencing factors.

The highest content in omega-3 fatty acids characterizes walnuts with respect to other nuts [36,37]. Although the impact of polyunsaturated fatty acids on the gut microbiota is poorly defined, several human studies have shown some common changes in the gut microbiota after omega-3 PUFA supplementation. In particular, a decrease in Faecalibacterium, from Ruminococcaceae family, often associated with an increase in other butyrate-producing bacteria belonging to the Lachnospiraceae family has been observed [38]. Walnuts present similarities in the chemical composition with other nuts, like almonds and pistachios, which do not contain ellagitannins. Some studies have investigated the effects of other dried fruits and nuts on the gut microbiota composition $[9,34,39]$. Ukhanova et al. demonstrated enrichment in butyrate producers after almonds and pistachios consumption for 18 days [34]. Holscher and colleagues performed an interventional study with almonds and found an increase in the relative abundances of several butyrate-producing bacteria such as Lachnospira, Roseburia, and other bacterial groups such as Dialister and Oscillospira [9]. In any case, all these studies focused on changes in gut microbial composition and agreed on the same point that these interventions produced an increase in butyrate-producing bacteria. In our study, a three-day walnut intervention was enough to increase acetic acid and propionate of fecal samples, without differences between UMs. Riviére et al. reported health benefits of propionate in mice by reducing serum cholesterol, improving insulin sensitivity, and promoting satiety [40]. Recently, the use of inulin-propionate ester, to deliver propionate to the colon selectively, improved insulin resistance in overweight and obese subjects [41]. 
A recent study (including 365 study participants in 13 trials) found that diets enriched with walnuts led to lower total and LDL-cholesterol when compared with other diets [42]. It has also been reported that walnut consumption is associated with a lower risk of type 2 diabetes [4]. The PREDIMED trial is the largest primary prevention trial showing that an intervention to promote a Mediterranean diet, which is beneficial against the incidence of several major chronic diseases in subjects at high cardiovascular risk, particularly when improved adherence to the Mediterranean diet includes increased consumption of extra virgin olive oil and mixed tree nuts. The PREDIMED trial showed that participants included in the MD group supplemented with nuts presented a lower risk of cardiovascular events and type 2 diabetes $[43,44]$.

The increase in acetate production is in accordance with the increase in the relative abundances of the Bifidobacterium genus. Moreover, acetate is used by cross-feeding species as a co-substrate to produce butyrate [40]. Therefore, this could mean that the increase of Bifidobacterium could favor the colonization of other beneficial butyrate-producing bacteria, such as Coprococcus, leading to an increase in butyrate production. Although we did not observe a significant increase in butyrate production, which could have been due to the short-term walnut intervention, we found positive correlations between higher acetate and propionate production, and Phascolarbacterium, Coprococcus, and Anaerostipes. These results agree with previous studies that described the production of SCFA, including acetate and propionate, by Phascolarctobacterium [45] as well as the production of butyrate by Coprococus and Anaerostipes [40]. At the species level, we also observed positive correlations between Coprococcus catus, butyrate, and propionate concentrations. This is related to the capability of this bacterial species for butyrate and propionate production, via butyryl-CoA: acetate CoA-transferase route and acrylate pathway, respectively [45]. It is well known that butyrate exerts health benefits, including the preservation of the gut barrier integrity. Remarkably, it has been demonstrated that urolithin-A (Uro-A), as a microbial metabolite, significantly enhances the gut barrier function through the up-regulation of the epithelial tight junctions' proteins [46], which confirmed pioneering investigations of our group that demonstrated for the first time the preservation of the colon architecture by Uro-A under intestinal inflammation [2]. Although fecal SCFA profiles are widely used as markers of gut health or disease, caution on interpreting the results may be required. More than $95 \%$ of SCFAs in the gut are quickly absorbed. Therefore, measurement of fecal SCFAs concentration only represents the $5 \%$ of produced SCFA left unabsorbed. Luminal concentrations of SCFAs can be affected by both rates of production and disappearance, circadian rhythms, section of the intestine, and specific microenvironment at luminal or mucosal compartments [47].

In summary, the main findings of this interventional study show that walnut consumption for three days is enough to modulate gut microbial composition in a UM-depending manner and to increase the production of SCFA. This study supports the concept of personalized nutrition and that not all the dietary recommendations may exert the same benefits in all individuals. This concept is growing and there is enough evidence that explains that a complex relationship between diet and microbiome could be behind this. Nevertheless, there are still difficulties predicting gut microbiota and host responses to a given dietary intervention. There is still no consensus because there are many parameters that we should take into account, such as host physiology, habitual dietary patterns, and gut microbiota baseline. Therefore, more research is needed and clinical trials should take into account this inter-individual variability.

Author Contributions: Conceptualization, M.V.S., J.C.E., and M.C.C.; methodology, software, and validation, I.G.-M., M.C., and M.R.-V.; original drafting, I.G.-M.; writing—review and editing, I.G.-M., M.C., M.V.S., J.C.E., and M.C.C.

Funding: This research was funded by the European Research Council under the European Union's Horizon 2020 Research and Innovation Program (ERC starting grant, $n^{\circ}$ 639226), the RTI2018-097982-B-I00 from the Spanish Ministry of Science, Innovation and Universities (MICINN) and AGL2015-64124-R (MINECO, Spain) and by the Research Foundation Flanders (postdoctoral fellowship FWO-12R2717N and travel grant FWO-V436918N).

Acknowledgments: Authors would acknowledge the support from the European Research Council under the European Union's Horizon 2020 Research and Innovation Program (ERC starting grant, $n^{\circ}$ 639226) and 
RTI2018-097982-B-I00 from the Spanish Ministry of Science, Innovation and Universities (MICINN) and AGL2015-64124-R (MINECO, Spain). M.C. is supported by the Research Foundation Flanders (postdoctoral fellowship FWO-12R2717N and travel grant FWO-V436918N). The authors also thank the participants involved in the study.

Conflicts of Interest: The authors declare no conflict of interest.

\section{Abbreviations}

$\begin{array}{ll}\text { GM } & \text { gut microbiota } \\ \text { UM } & \begin{array}{l}\text { urolithin metabotype } \\ \text { real-time quantitative polymerase chain reaction } \\ \text { qPCR }\end{array} \\ \text { ultra-high performance liquid chromatography-quadrupole time-of-flight mass } \\ \text { spectrometry } \\ \text { ellagitannins } \\ \text { ETs } & \text { ellagic acid } \\ \text { EA } & \text { urolithin } \\ \text { Uro } & \text { isourolithin } \\ \text { IsoUro } & \text { cardiovascular disease } \\ \text { CVD } & \text { short chain fatty acids } \\ \text { SCFA } & \text { body mass index } \\ \text { BMI } & \text { food frequency questionnaire } \\ \text { FFQ } & \text { Centro de Enseñanza Superior de Nutrición Humana y Dietética } \\ \text { CESNID } & \text { PREvención con DIeta MEDiterránea } \\ \text { PREDIMED } & \text { linear discriminant analysis effect size } \\ \text { LEfSe } & \text { redundancy analysis } \\ \text { RDA } & \end{array}$

\section{References}

1. Ros, E. Health benefits of nut consumption. Nutrients 2010, 2, 652-682. [CrossRef] [PubMed]

2. Larrosa, M.; González-Sarrías, A.; Yáñez-Gascón, M.J.; Selma, M.V.; Azorín-Ortuño, M.; Toti, S.; Tomás-Barberán, F.; Dolara, P.; Espín, J.C. Anti-inflammatory properties of a pomegranate extract and its metabolite urolithin-A in a colitis rat model and the effect of colon inflammation on phenolic metabolism. J. Nutr. Biochem. 2010, 21, 717-725. [CrossRef] [PubMed]

3. Selma, M.V.; Espín, J.C.; Tomás-Barberán, F.A. Interaction between Phenolics and Gut Microbiota: Role in Human Health. J. Agric. Food Chem. 2009, 57, 6485-6501. [CrossRef] [PubMed]

4. Salas-Salvadó, J.; Bulló, M.; Pérez-Heras, A.; Ros, E. Dietary fibre, nuts and cardiovascular diseases. Br. J. Nutr. 2006, 96, S45-S51. [CrossRef]

5. Cardona, F.; Andrés-Lacueva, C.; Tulipani, S.; Tinahones, F.J.; Queipo-Ortuño, M.I. Benefits of polyphenols on gut microbiota and implications in human health. J. Nutr. Biochem. 2013, 24, 1415-1422. [CrossRef]

6. González-Sarrías, A.; García-Villalba, R.; Romo-Vaquero, M.; Alasalvar, C.; Örem, A.; Zafrilla, P.; Tomás-Barberán, F.A.; Selma, M.V.; Espín, J.C. Clustering according to urolithin metabotype explains the interindividual variability in the improvement of cardiovascular risk biomarkers in overweight-obese individuals consuming pomegranate: A randomized clinical trial. Mol. Nutr. Food Res. 2017, 61, 1600830. [CrossRef]

7. Byerley, L.O.; Samuelson, D.; Blanchard, E.; Luo, M.; Lorenzen, B.N.; Banks, S.; Ponder, M.A.; Welsh, D.A.; Taylor, C.M. Changes in the gut microbial communities following addition of walnuts to the diet. J. Nutr. Biochem. 2017, 48, 94-102. [CrossRef]

8. Holscher, H.D.; Guetterman, H.M.; Swanson, K.S.; An, R.; Matthan, N.R.; Lichtenstein, A.H.; Novotny, J.A.; Baer, D.J. Walnut Consumption Alters the Gastrointestinal Microbiota, Microbially Derived Secondary Bile Acids, and Health Markers in Healthy Adults: A Randomized Controlled Trial. J. Nutr. 2018, 148, 861-867. [CrossRef]

9. Holscher, H.D.; Taylor, A.M.; Swanson, K.S.; Novotny, J.A.; Baer, D.J. Almond Consumption and Processing Affects the Composition of the Gastrointestinal Microbiota of Healthy Adult Men and Women: A Randomized Controlled Trial. Nutrients 2018, 10, 126. [CrossRef] 
10. Han, Y.; Song, M.; Gu, M.; Ren, D.; Zhu, X.; Cao, X.; Li, F.; Wang, W.; Cai, X.; Yuan, B.; et al. Dietary Intake of Whole Strawberry Inhibited Colonic Inflammation in Dextran-Sulfate-Sodium-Treated Mice via Restoring Immune Homeostasis and Alleviating Gut Microbiota Dysbiosis. J. Agric. Food Chem. 2019. [CrossRef]

11. Tomás-Barberán, F.A.; García-Villalba, R.; González-Sarrías, A.; Selma, M.V.; Espín, J.C. Ellagic Acid Metabolism by Human Gut Microbiota: Consistent Observation of Three Urolithin Phenotypes in Intervention Trials, Independent of Food Source, Age, and Health Status. J. Agric. Food Chem. 2014, 62, 6535-6538. [CrossRef] [PubMed]

12. Tomás-Barberán, F.A.; González-Sarrías, A.; García-Villalba, R.; Núñez-Sánchez, M.A.; Selma, M.V.; García-Conesa, M.T.; Espín, J.C. Urolithins, the rescue of "old" metabolites to understand a "new" concept: Metabotypes as a nexus among phenolic metabolism, microbiota dysbiosis, and host health status. Mol. Nutr. Food Res. 2017, 61, 1500901. [CrossRef] [PubMed]

13. Beltrán, D.; Romo-Vaquero, M.; Espín, J.C.; Tomás-Barberán, F.A.; Selma, M.V. Ellagibacter isourolithinifaciens gen. nov., sp. nov., a new member of the family Eggerthellaceae, isolated from human gut. Int. J. Syst. Evol. Microbiol. 2018, 68, 1707-1712. [CrossRef] [PubMed]

14. Selma, M.V.; Beltrán, D.; García-Villalba, R.; Espín, J.C.; Tomás-Barberán, F.A. Description of urolithin production capacity from ellagic acid of two human intestinal Gordonibacter species. Food Funct. 2014, 5, 1779-1784. [CrossRef]

15. Selma, M.V.; Tomas-Barberan, F.A.; Beltran, D.; Garcia-Villalba, R.; Espin, J.C. Gordonibacter urolithinfaciens sp. nov., a urolithin-producing bacterium isolated from the human gut. Int. J. Syst. Evol. Microbiol. 2014, 64, 2346-2352. [CrossRef]

16. Selma, M.V.; Beltrán, D.; Luna, M.C.; Romo-Vaquero, M.; García-Villalba, R.; Mira, A.; Espín, J.C.; Tomás-Barberán, F.A. Isolation of Human Intestinal Bacteria Capable of Producing the Bioactive Metabolite Isourolithin A from Ellagic Acid. Front. Microbiol. 2017, 8, 1521. [CrossRef]

17. Selma, M.V.; González-Sarrías, A.; Salas-Salvadó, J.; Andrés-Lacueva, C.; Alasalvar, C.; Örem, A.; Tomás-Barberán, F.A.; Espín, J.C. The gut microbiota metabolism of pomegranate or walnut ellagitannins yields two urolithin-metabotypes that correlate with cardiometabolic risk biomarkers: Comparison between normoweight, overweight-obesity and metabolic syndrome. Clin. Nutr. 2018, 37, 897-905. [CrossRef]

18. Cortés-Martín, A.; Selma, M.V.; Espín, J.C.; García-Villalba, R. The Human Metabolism of Nuts Proanthocyanidins does not Reveal Urinary Metabolites Consistent with Distinctive Gut Microbiota Metabotypes. Mol. Nutr. Food Res. 2019, 63, 1800819. [CrossRef]

19. García-Villalba, R.; Espín, J.C.; Aaby, K.; Alasalvar, C.; Heinonen, M.; Jacobs, G.; Voorspoels, S.; Koivumäki, T.; Kroon, P.A.; Pelvan, E.; et al. Validated Method for the Characterization and Quantification of Extractable and Nonextractable Ellagitannins after Acid Hydrolysis in Pomegranate Fruits, Juices, and Extracts. J. Agric. Food Chem. 2015, 63, 6555-6566. [CrossRef]

20. Salas-Salvadó, J.; Rubio, M.A.; Barbany, M.; Moreno, B. Consenso SEEDO 2007 para la evaluación del sobrepeso y la obesidad y el establecimiento de criterios de intervención terapéutica. Conferencia de consenso. Med. Clin. 2007, 128, 184-196.

21. Fernández-Ballart, J.D.; Piñol, J.L.; Zazpe, I.; Corella, D.; Carrasco, P.; Toledo, E.; Perez-Bauer, M.; Martínez-González, M.Á.; Salas-Salvadó, J.; Martín-Moreno, J.M. Relative validity of a semi-quantitative food-frequency questionnaire in an elderly Mediterranean population of Spain. Br. J. Nutr. 2010, 103, 1808-1816. [CrossRef] [PubMed]

22. Martinez-Gonzalez, M.A.; Corella, D.; Salas-Salvado, J.; Ros, E.; Covas, M.I.; Fiol, M.; Warnberg, J.; Aros, F.; Ruiz-Gutierrez, V.; Lamuela-Raventos, R.M.; et al. Cohort Profile: Design and methods of the PREDIMED study. Int. J. Epidemiol. 2012, 41, 377-385. [CrossRef] [PubMed]

23. García-Villalba, R.; Espín, J.C.; Tomás-Barberán, F.A. Chromatographic and spectroscopic characterization of urolithins for their determination in biological samples after the intake of foods containing ellagitannins and ellagic acid. J. Chromatogr. A 2016, 1428, 162-175. [CrossRef] [PubMed]

24. Cortés-Martín, A.; García-Villalba, R.; González-Sarrías, A.; Romo-Vaquero, M.; Loria-Kohen, V.; Ramírez-de-Molina, A.; Tomás-Barberán, F.A.; Selma, M.V.; Espín, J.C. The gut microbiota urolithin metabotypes revisited: The human metabolism of ellagic acid is mainly determined by aging. Food Funct. 2018, 9, 4100-4106. [CrossRef] 
25. Garcia-Mantrana, I.; Selma-Royo, M.; Alcantara, C.; Collado, M.C. Shifts on Gut Microbiota Associated to Mediterranean Diet Adherence and Specific Dietary Intakes on General Adult Population. Front. Microbiol. 2018, 9, 890. [CrossRef]

26. Romo-Vaquero, M.; García-Villalba, R.; González-Sarrías, A.; Beltrán, D.; Tomás-Barberán, F.A.; Espín, J.C.; Selma, M.V. Interindividual variability in the human metabolism of ellagic acid: Contribution of Gordonibacter to urolithin production. J. Funct. Foods 2015, 17, 785-791. [CrossRef]

27. Caporaso, J.G.; Kuczynski, J.; Stombaugh, J.; Bittinger, K.; Bushman, F.D.; Costello, E.K.; Fierer, N.; Peña, A.G.; Goodrich, J.K.; Gordon, J.I.; et al. QIIME allows analysis of high-throughput community sequencing data. Nat. Methods 2010, 7, 335-336. [CrossRef]

28. Segata, N.; Izard, J.; Waldron, L.; Gevers, D.; Miropolsky, L.; Garrett, W.S.; Huttenhower, C. Metagenomic biomarker discovery and explanation. Genome Biol. 2011, 12, R60. [CrossRef]

29. Andersen, S.J.; Hennebel, T.; Gildemyn, S.; Coma, M.; Desloover, J.; Berton, J.; Tsukamoto, J.; Stevens, C.; Rabaey, K. Electrolytic Membrane Extraction Enables Production of Fine Chemicals from Biorefinery Sidestreams. Environ. Sci. Technol. 2014, 48, 7135-7142. [CrossRef]

30. Espín, J.C.; González-Sarrías, A.; Tomás-Barberán, F.A. The gut microbiota: A key factor in the therapeutic effects of (poly)phenols. Biochem. Pharmacol. 2017, 139, 82-93. [CrossRef]

31. Healey, G.R.; Murphy, R.; Brough, L.; Butts, C.A.; Coad, J. Interindividual variability in gut microbiota and host response to dietary interventions. Nutr. Rev. 2017, 75, 1059-1080. [CrossRef] [PubMed]

32. Romo-Vaquero, M.; Cortés-Martín, A.; Loria-Kohen, V.; Ramírez-de-Molina, A.; García-Mantrana, I.; Collado, M.C.; Espín, J.C.; Selma, M.V. Deciphering the Human Gut Microbiome of Urolithin Metabotypes: Association with Enterotypes and Potential Cardiometabolic Health Implications. Mol. Nutr. Food Res. 2019, 63, 1800958. [CrossRef] [PubMed]

33. Tulipani, S.; Urpi-Sarda, M.; García-Villalba, R.; Rabassa, M.; López-Uriarte, P.; Bulló, M.; Jáuregui, O.; Tomás-Barberán, F.; Salas-Salvadó, J.; Espín, J.C.; et al. Urolithins Are the Main Urinary Microbial-Derived Phenolic Metabolites Discriminating a Moderate Consumption of Nuts in Free-Living Subjects with Diagnosed Metabolic Syndrome. J. Agric. Food Chem. 2012, 60, 8930-8940. [CrossRef] [PubMed]

34. Ukhanova, M.; Wang, X.; Baer, D.J.; Novotny, J.A.; Fredborg, M.; Mai, V. Effects of almond and pistachio consumption on gut microbiota composition in a randomised cross-over human feeding study. Br. J. Nutr. 2014, 111, 2146-2152. [CrossRef] [PubMed]

35. Bamberger, C.; Rossmeier, A.; Lechner, K.; Wu, L.; Waldmann, E.; Fischer, S.; Stark, R.; Altenhofer, J.; Henze, K.; Parhofer, K. A Walnut-Enriched Diet Affects Gut Microbiome in Healthy Caucasian Subjects: A Randomized, Controlled Trial. Nutrients 2018, 10, 244. [CrossRef] [PubMed]

36. Ros, E.; Mataix, J. Fatty acid composition of nuts-implications for cardiovascular health. Br. J. Nutr. 2006, 96, S29-S35. [CrossRef]

37. Lamuel-Raventos, R.M.; Onge, M.-P.S. Prebiotic nut compounds and human microbiota. Crit. Rev. Food Sci. Nutr. 2017, 57, 3154-3163. [CrossRef]

38. Costantini, L.; Molinari, R.; Farinon, B.; Merendino, N. Impact of Omega-3 Fatty Acids on the Gut Microbiota. Int. J. Mol. Sci. 2017, 18, 2645. [CrossRef]

39. Burns, A.M.; Zitt, M.A.; Rowe, C.C.; Langkamp-Henken, B.; Mai, V.; Nieves, C.; Ukhanova, M.; Christman, M.C.; Dahl, W.J. Diet quality improves for parents and children when almonds are incorporated into their daily diet: A randomized, crossover study. Nutr. Res. 2016, 36, 80-89. [CrossRef]

40. Rivière, A.; Selak, M.; Lantin, D.; Leroy, F.; De Vuyst, L. Bifidobacteria and Butyrate-Producing Colon Bacteria: Importance and Strategies for Their Stimulation in the Human Gut. Front. Microbiol. 2016, 7, 979. [CrossRef]

41. Chambers, E.S.; Byrne, C.S.; Morrison, D.J.; Murphy, K.G.; Preston, T.; Tedford, C.; Garcia-Perez, I.; Fountana, S.; Serrano-Contreras, J.I.; Holmes, E.; et al. Dietary supplementation with inulin-propionate ester or inulin improves insulin sensitivity in adults with overweight and obesity with distinct effects on the gut microbiota, plasma metabolome and systemic inflammatory responses: A randomised cross-over trial. Gut 2019, 68, 1430-1438. [PubMed]

42. Banel, D.K.; Hu, F.B. Effects of walnut consumption on blood lipids and other cardiovascular risk factors: A meta-analysis and systematic review. Am. J. Clin. Nutr. 2009, 90, 56-63. [CrossRef] [PubMed] 
43. Martinez-Lapiscina, E.H.; Clavero, P.; Toledo, E.; San Julian, B.; Sanchez-Tainta, A.; Corella, D.; Lamuela-Raventos, R.M.; Martinez, J.A.; Martinez-Gonzalez, M.Á. Virgin olive oil supplementation and long-term cognition: The Predimed-Navarra randomized, trial. J. Nutr. Health Aging 2013, 17, 544-552. [CrossRef] [PubMed]

44. Estruch, R.; Ros, E.; Salas-Salvadó, J.; Covas, M.-I.; Corella, D.; Arós, F.; Gómez-Gracia, E.; Ruiz-Gutiérrez, V.; Fiol, M.; Lapetra, J.; et al. Primary Prevention of Cardiovascular Disease with a Mediterranean Diet. N. Engl. J. Med. 2013, 368, 1279-1290. [CrossRef] [PubMed]

45. Louis, P.; Flint, H.J. Formation of propionate and butyrate by the human colonic microbiota. Environ. Microbiol. 2017, 19, 29-41. [CrossRef] [PubMed]

46. Singh, R.; Chandrashekharappa, S.; Bodduluri, S.R.; Baby, B.V.; Hegde, B.; Kotla, N.G.; Hiwale, A.A.; Saiyed, T.; Patel, P.; Vijay-Kumar, M.; et al. Enhancement of the gut barrier integrity by a microbial metabolite through the Nrf2 pathway. Nat. Commun. 2019, 10, 89. [CrossRef]

47. Sakata, T. Pitfalls in short-chain fatty acid research: A methodological review. Anim. Sci. J. 2019, 90, 3-13. [CrossRef]

(C) 2019 by the authors. Licensee MDPI, Basel, Switzerland. This article is an open access article distributed under the terms and conditions of the Creative Commons Attribution (CC BY) license (http://creativecommons.org/licenses/by/4.0/). 